\title{
Nurses' experiences of communication during the coronavirus disease 2019 pandemic: A qualitative study
}

\author{
Zahra Khademi $^{\circledR}$, Elham Imani ${ }^{2^{*}}$, Mohamad Heydari Khormizi ${ }^{3^{\circledR}}$ \\ 'MSc in Nursing, Department of Nursing, Faculty of Nursing and Midwifery, Hormozgan University of Medical Sciences, \\ Bandar Abbas, Iran \\ ${ }^{2}$ Infectious and Tropical Diseases Research Center, Hormozgan Health Institute, Hormozgan University of Medical Sciences, \\ Bandar Abbas, Iran \\ ${ }^{3}$ MSc Student in Clinical Psychology, Bandar Abbas, Iran
}

\begin{abstract}
Background and aims: Effective communication is a key to quality nursing care delivery. However, the necessity to wear personal protective equipment and keep physical distancing during the coronavirus disease 2019 (COVID-19) pandemic has created numerous challenges in establishing effective communication in healthcare settings. The aim of this study was to qualitatively explore nurses' experiences of communication during the COVID-19 pandemic.

Methods: This qualitative study was conducted in 2020 using the Van Manen's hermeneutic or interpretational phenomenological design. Participants were thirteen nurses purposively selected from the COVID-19 care ward of a teaching hospital in Bandar Abbas, Iran. Data were collected through semi-structured interviews and were analyzed using the Van Manen's approach to phenomenological research.

Results: Most participants were female $(69.2 \%)$ and had bachelor's degree $(84.6 \%)$. The means of their age and work experience were $32.08 \pm 5.42$ and $9.54 \pm 5.22$ years, respectively. A total of 1312 codes were generated during data analysis, which were grouped into 28 subcategories, thirteen categories, and the four themes of being in dire straits, social life disturbances, disturbance of peace, and ray of hope.

Conclusion: The COVID-19 pandemic has challenged nurses' communication and hence, they need emotional and psychological support to remain hopeful. Nursing managers need to support nurses and remove barriers to their effective communication in order to enable them to provide safe and quality care. Keywords: Communication, Coronavirus disease 2019, Nurse, Qualitative study
\end{abstract}

*Corresponding Author: Elham Imani, PhD, Infectious and Tropical Diseases Research Center, Hormozgan Health Institute, Hormozgan University of Medical Sciences, Bandar Abbas, Iran. Tell: 076-33346994; Fax: 076-33346994. Email: eimani@hums.ac.ir

Received: 17 April 2021 Accepted: 28 July 2021 ePublished: 30 September 2021

\section{Introduction}

Communication is a key to the success of healthcare teams (1) and the most basic prerequisite to care delivery to healthcare clients (2). It is a multidimensional and multifactorial phenomenon and a dynamic and complex process, without which individuals cannot show their concerns, feel what is happening to them, and link themselves to the environment (1). Effective communication keeps individuals close together and helps them understand others' feelings (2).

Nurses are healthcare providers with the highest level of communication in healthcare settings. They spend most of their time in direct contact with physicians, nutrition specialists, physiotherapists, patients, patients' family members, and many other people during their daily practice. Moreover, as patients experience high levels of stress due to hospitalization and fear over the unknown, nurses should communicate with them to provide them with the necessary health-related information (3) and fulfill their needs (1). Communication in healthcare settings is established through speaking, body language, listening, writing, reading, and information technologies such as electronic medical records and boards (3).
Epidemics, such as the coronavirus disease 2019 (COVID-19) pandemic, disturb daily activities, require physical distancing to reduce the risk of infection transmission (4), require healthcare providers to use personal protective equipment (5), and thereby, significantly affect all aspects of human life (1), including communication (5). While patients need nurses' nonverbal behaviors, particularly when they have anxiety or uncertainty (1), face masks and shields disturb verbal and nonverbal communication and reduce the ability to use facial expressions (5). Because of communication limitation during the COVID-19 pandemic, many patients have to communicate with their significant others through telephone or with plastic gowns and masks (6).

Poor communication in healthcare settings is associated with different adverse consequences. For example, limitation of patient visitation by family members during the COVID-19 pandemic has resulted in the sense of social isolation for patients and their family members (5) and the deaths of patients in loneliness (6). A study reported that separation from the beloved ones was associated with high levels of stress and fear for patients with COVID-19 (7). Patients with COVID-19 are also unable to clearly see 
nurses and hear their voices because nurses in healthcare settings should use personal protective equipment (8). A study during Middle East Respiratory Syndrome (MERS) reported communication disturbances as a leading cause of restlessness and stress among healthcare providers (4). Another study reported a significant negative relationship between patients' perception of empathy and physicians' use of face mask during patient visit (5). Failure in communication can also lead to mistrust, negative financial effects, and even death (9). Isolation of patients with COVID-19 in single-bed rooms in hospitals also brings them senses of boredom and social isolation (10), deprives them of family support, and puts their family members at risk for posttraumatic stress disorder and grief (11).

The COVID-19 pandemic has also negatively affected nurses' communication with their families, peers, colleagues, and society. A study showed that in the early days of the COVID-19 pandemic, nurses in Wuhan, China, who were not originally from Wuhan, were unable to communicate with people out of Wuhan and hence, felt lonely (12). Another study reported that 29\% of the members of a Chinese anti-Ebola care delivery team suffered from loneliness (13). Fear over infection transmission to family members separates nurses from their families, makes them homesick for their families, and increases their need for family members' kindness (14). Moreover, use of face mask and limitation of communication among different healthcare providers can negatively affect their relationships and cause them depersonalization (5). A study into the experiences of nurses during the MERS epidemic and the COVID-19 pandemic indicated that care delivery to critically-ill patients in isolation rooms seriously limited nurses' communication with the outside environment and their colleagues, caused their non-nurse colleagues to distance from them, gave them senses of loneliness and abandonment, and caused social discrimination of nurses such as limited nursery services for their children (15). Another study reported moral judgments and social stigmatization as experiences of nurses in their social relationships during the COVID-19 pandemic (8). A study also reported that the temporary suppression of the needs for interpersonal relationships, kindness, safety, and health-related knowledge due to epidemics increases these needs among nurses and causes them high levels of stress (14).

Despite the wealth of studies into communication, there is limited information about communication during epidemics, particularly in Iran. Therefore, the present study was designed and conducted to narrow this gap. The aim of the study was to qualitatively explore nurses' experiences of communication during the COVID-19 pandemic.

\section{Methods}

Design
This qualitative study was conducted in 2020 using the hermeneutic or interpretational phenomenological design recommended by Van Manen. Phenomenology is the study of the personal world, i.e., the world each individual experiences prior to thinking about it or conceptualizing it, without any prejudice or theoretical ideas. Accordingly, phenomenological researchers attempt to put aside their prejudices and experiences and rely on intuition to achieve an accurate understanding of their intended phenomena (16). Phenomenological designs are appropriate for the in-depth exploration of the different aspects of lived experiences and poorly known phenomena such as communication during the COVID-19 pandemic. In phenomenological studies, researchers attempt to enter the world of participants and explore their lived experiences. The hermeneutic or interpretational phenomenological design focuses on the understanding and interpretation of human experiences and aims at entering and discovering the real world of people (17). The Van Manen's phenomenological approach presents a systematic approach to study and interpret poorly known phenomena (18).

\section{Participants and setting}

Study participants were nurses purposively selected from the COVID-19 care ward of Shahid Mohammadi hospital, Bandar Abbas, Iran. This teaching hospital is affiliated to Hormozgan University of Medical Sciences, Bandar Abbas, Iran. Inclusion criteria were employment as nurse in COVID-19 care ward and agreement for participation.

\section{Data collection}

Data were collected via face-to-face semi-structured interviews guided by an interview guide with broad questions such as, "Can you please explain about communication with patients with COVID-19?" "What are your experiences of COVID-19?" "Can you provide an example of your best experiences during the COVID-19 pandemic?" and "Can you provide an example of your worst experiences during the COVID-19 pandemic?" At the beginning of the interviews, questions about participants' demographic characteristics were used and then, the main interview questions were asked. Each interview was continued until the interviewer was ensured about the full exploration of the interviewee's experiences of communication in the COVID-19 pandemic. Interviews lasted 40-70 minutes with a mean of 54 minutes. Data collection was continued up to data saturation, i.e., when no new data were obtained from the interviews. All interviews were audio recorded. Immediately after each interview, it was transcribed word by word for analysis.

\section{Data analysis}

Interview data were analyzed using Van Manen's approach to phenomenological research. The six steps of this approach are assessment of and encounter with the nature of the experience, in-depth assessment of the phenomenon, 
reflection on the essential themes of the phenomenon, interpretational writing and rewriting, maintenance of a strong and oriented relation with the phenomenon, and creation of a coherence through considering the whole phenomenon and its parts. Participants' demographic characteristics were described through the measures of descriptive statistics.

\section{Trustworthiness}

The trustworthiness of the study was confirmed through the four criteria of confirmability, credibility, dependability, and transferability recommended by Lincoln and Guba. In order to receive confirmability, the researchers take to expand data validity by increasing the number of interviews. The researcher tried to make more close relationship with the participants. Credibility was confirmed through member checking, in which some participants assessed and confirmed the congruence between our findings and their own experiences. Then, dependability was ensured through external peer checking by several qualitative researchers. Transferability was also ensured through purposive sampling with maximum variation.

\section{Results}

Participants were thirteen nurses selected from COVID-19 care ward of a teaching hospital in Iran. They were mostly female (69.2\%), had bachelor's degree (84.6\%), and the means of their age and work experience were 32.08 \pm 5.42 and $9.54 \pm 5.22$ years, respectively (Table 1 ).

A total of 1312 codes were generated during data analysis, which were grouped into 28 subcategories, 13 categories, and four themes (Table 2). The four themes were being in dire straits, social life disturbances, disturbance of peace, and ray of hope. The COVID-19 pandemic has altered social relationships and its associated restrictions have given a sense of dire straits to nurses. Moreover, it has changed public attitude towards the relatives of patients

Table 1. Participants' demographic characteristics

\begin{tabular}{|c|c|c|c|}
\hline Characteristics & Groups & $\mathbf{N}$ & $\%$ \\
\hline \multirow{2}{*}{ Gender } & Male & 4 & 30.8 \\
\hline & Female & 9 & 69.2 \\
\hline \multirow{2}{*}{ Educational level } & Bachelor's degree & 11 & 84.6 \\
\hline & Master's degree & 2 & 15.4 \\
\hline \multirow[t]{2}{*}{ Total } & & 13 & 100 \\
\hline & Minimum & & \\
\hline \multirow[t]{2}{*}{ Age $(y)$} & Maximum & & \\
\hline & Mean \pm SD & & \\
\hline \multirow{3}{*}{ Work experience $(y)$} & Minimum & & \\
\hline & Maximum & & \\
\hline & Mean \pm SD & & \\
\hline \multirow{3}{*}{ Length of interview (min) } & Minimum & & \\
\hline & Maximum & & \\
\hline & Mean \pm SD & & \\
\hline
\end{tabular}

who die of COVID-19 and prevents these relatives from having a normal grief which in turn disturbs their social life. Healthcare providers also have physical and mental tensions due to the pandemic and need to wear personal protective equipment which pose them considerable difficulties and disturb their peace. The only ray of hope for them in such difficult situations is patients' kind sayings and people's satisfaction with their services.

\section{Being in dire straits}

The necessity of physical distancing, limitation of social and familial relationships, reduction of nurses' presence in family circles, and some individuals' distancing from nurses due to their fear over COVID-19 transmission have given nurses unpleasant feelings such as isolation and emotional deprivation and caused them limitations in their communications and daily activities. Moreover, the necessity to use face mask has negatively affected their ability to establish nonverbal communication.

"Our responsibility towards patients on the one hand and our fear over transmitting COVID-19 to our families on the other hand have imposed heavy stress on us" (P. 11).

"Nurses have become really isolated. They fear from attending the society because people think that they are COVID-19 carrier" (P. 8).

"It is now for twelve days that I haven't been home. I'm really homesick for my daughter. I have fear over transmitting COVID-19 to my family and my child. All of us have fear over being COVID-19 carrier" (P. 10).

"Observing the restrictions is the most effective strategy to control this epidemic. All of us should observe physical distancing and limit our relationships. Most afflicted patients are those who don't observe restrictions and have not reduced their family relationships" (P. 7).

Communication has become very difficult in the current conditions. Because of using protective equipment, we can just see each other's eyes. It is very difficult to understand our colleagues' emotions from their eyes" (P. 12).

Table 2. The themes and categories of nurses' experiences of communication during the COVID-19 pandemic

\begin{tabular}{|c|c|}
\hline Themes & Categories \\
\hline \multirow{4}{*}{ Being in dire straits } & Feeling isolated \\
\hline & Emotional deprivation \\
\hline & Restrictions of daily activities \\
\hline & Alteration of nonverbal communication \\
\hline \multirow{3}{*}{ Social life disturbances } & Isolation \\
\hline & Social stigmatization \\
\hline & Disturbed mourning \\
\hline \multirow{3}{*}{ Disturbance of peace } & Personal protective equipment \\
\hline & Negative thoughts \\
\hline & Physical tensions \\
\hline \multirow{3}{*}{ Ray of hope } & Public appreciation \\
\hline & Empathy \\
\hline & Receiving positive energy \\
\hline
\end{tabular}




\section{Social life disturbances}

Some individuals have not still accepted COVID-19 as a communicable disease which should be considered as a part of life like flu. Consequently, afflicted patients are socially isolated and stigmatized even after complete recovery. Such isolation and stigmatization disturb recovered patients' lives and give them unpleasant feelings. On the other hand, the high risk of COVID-19 affliction prevents the family members of died patients from having a normal grief process which in turn causes conflicts in their relationships.

"We had a 70-year-old male patient who died due to COVID-19. Unfortunately, his family members abandoned him and escaped from the hospital as soon as they noticed his affliction by COVID-19" (P. 9).

"One of our relatives died due to COVID-19. The heads of the family had difficulties in accepting not to hold mourning ceremony and said that it was impossible not to hold the ceremony and not to invite people to the ceremony. It was very difficult to satisfy them to do something else such as giving a charity for the passed patient instead of holding a ceremony" (P. 11).

\section{Disturbance of peace}

The necessity to use personal protective equipment, the difficulty of removing them, and the thirst, hunger, fatigue, warmth, and difficulty of breathing when using them are among the factors which disturb nurses' peace and comfort. Moreover, disturbing thoughts due to the fear over affliction by COVID-19 or attributing any symptom to COVID-19 cause nurses negative feelings.

"We had to closely adhere to the precautions during care delivery to these patients. However, wearing gown and mask and not drinking water and eating food for eight hours are very difficult and torturing" (P. 9).

"I constantly think that I'm afflicted by COVID-19. I have a constant feeling of dyspnea and have symptoms which worry me over affliction by COVID-19 despite I have been tested negative for COVID-19" (P. 3).

"Wearing these clothes in the winter was easy; however, they are now intolerable in this warm weather. Working with these clothes is very difficult because I become wholly soaked in sweat with them" (P. 6).

\section{Ray of hope}

Despite all difficulties in communicating with patients and colleagues, factors such as the recovered patients' gratitude and public appreciation for nurses motivate them and give them energy for work. Moreover, satisfaction of government authorities with nurses' performance has significant role in motivating nurses for care delivery.

"My best memory is the satisfaction of COVID-19afflicted patients with our services. Their sentences calm me" (P. 4).

"Specifically, the leader had a sweet talk for thanking nurses which was greatly calming. All of us felt great energy due to his talk. His remembering of us and appreciation of our activities are very valuable" (P. 5)

"Although we face different difficulties and have a frightening appearance [due to personal protective equipment], we need to kindly treat patients because they are isolated and have no visitation by family members" (P. 11).

\section{Discussion}

This study explored nurses' experiences of communication during the COVID-19 pandemic. Findings revealed that the four main themes of nurses' experiences of communication during the COVID-19 pandemic were being in dire straits, social life disturbances, disturbance of peace, and ray of hope.

The first main theme of the study was being in dire straits. The COVID-19 pandemic has substantially limited interpersonal relationships and interpersonal understanding, particularly among healthcare providers who are in direct contact with the disease and the virus. Such limitation has caused nurses problems such as social isolation, emotional deprivation, problems in daily activities and communications, and thereby, has placed nurses in dire straits. Two previous studies on healthcare providers in COVID-19 care wards also reported feelings of loneliness and isolation $(19,20)$. Another study on healthcare providers in COVID-19 care wards in China found that the prevalence of depression was $45.6 \%$ among physicians and $43 \%$ among nurses (21). Similarly, a study reported high levels of anxiety and psychological trauma during and after care delivery to patients with severe acute respiratory syndrome and high prevalence of posttraumatic disorder and high levels of psychological needs among nurses who provided care to patients with MERS (15). Physical distancing and use of personal protective equipment to prevent COVID-19 transmission cause challenges in effective verbal and nonverbal communication between healthcare providers and clients (5). While nonverbal communication can improve patients' engagement in care and satisfaction with care, using face mask reduces the ability to see the whole face and express and detect emotions (22). On the other hand, inability to establish nonverbal communication and see the face of colleagues causes nurses problems such as burnout and dissociation from other healthcare members (23).

Like other people, nurses have social needs and psychological support can help them better cope with their conditions. Because of the impairment of face-toface communication during the COVID-19 pandemic, alternative methods of interpersonal communications should be considered. Examples of these methods are encouraging nurses during work shifts and printing congratulation and happy messages on personal protective equipment (14).

Social life disturbance was the second main theme of the study. Study findings revealed that affliction by COVID-19 negatively affects the life of patients and causes them loneliness even after recovery because 
people stigmatize them and avoid direct relationships with them. A previous study also revealed that nurses afflicted by MERS suffered from negative public attitude towards them, felt discrimination, and did not accept people's attitudes towards afflicted patients (15). A study reported that one of the ethical challenges of COVID-19 for nurses was witnessing the death of patients who experienced death in the absence of their beloved ones (6). Distancing between patients and families is also a major challenge during the COVID-19 pandemic which lasts even after death because family members neither can attend patient bedside during the process of dying, nor can hold mourning ceremony after their patient death due to the necessity of physical distancing. The necessity of physical distancing in hospital settings to reduce the risk of COVID-19 transmission gives patients and their families senses of loneliness and isolation and disturbs families' relationships with healthcare providers and hence, healthcare providers cannot effectively inform families of their patients' conditions. Moreover, ineffective communication between healthcare providers and clients during the COVID-19 pandemic has altered hospital discharge plans and educations and increased the risk of errors and safety events due to healthcare clients' poor understanding of educational materials.

The third main theme of the study was disturbance of nurses' peace due to the need for using personal protective equipment, physical tensions, and negative thoughts about affliction by COVID-19. Findings showed that these problems negatively affect nurses' communication with patients and colleagues. In line with this finding, a previous study reported nurses' increased vulnerability during care delivery to patients with COVID-19 due to their constant worry over personal health and COVID-19 transmission to their families as well as nurses' physical and mental problems during the MERS outbreak due to the difficulty of using personal protective equipment (19). A study into the sources and the symptoms of stress among nurses in a Chinese anti-Ebola medical team reported that the main sources of stress were safety concerns, accountability, and limited information about patients while the major symptoms of stress were sleep disorders, physiological problems, cognitive and emotional disorders (such as anxiety, mood swings, negative thoughts, attention deficit, and memory impairment), and behavioral problems (24). Strong support by family members, peers, and nursing managers are essential to reduce nurses' stress and improve the quality of their care services.

Ray of hope was the last main theme of the study. In epidemics, negative emotions overwhelm nurses and hence, nurses' self-reflection on their values and dedications and public appreciation of their performance can facilitate their personal and professional promotion and give them positive feelings (13). Fulfillment of their psychological needs through professional psychological support by specialized support groups can maintain their health during epidemics (14). Family and peer support can also have positive effects on nurses' health. A study reported that poor social support by family members, peers, and managers has significant relationship with nurses' burnout during epidemics (15). Therefore, strong support by families, peers, and managers for nurses through strategies such as sending them birthday messages and providing them with the opportunity to communicate with their families through video calls can reduce nurses' worry, boost their morale, and give them hope (24). All infectious diseases which require isolation can negatively affect communications among nurses, patients, and families. Thus, effective supportive strategies such as managerial and public appreciation are needed to give hope to nurses in order to protect them against job burnout and boost their morale.

\section{Conclusion}

This study suggests that in the COVID-19 pandemic, nurses experience dare straits, social life disturbances, and disturbance of peace during their communication and need strong family, peer, and managerial support and public appreciation in order to protect and promote their health. Therefore, psychoemotional support by managers is needed to boost their hope, manage their communication challenges and problems, remove barriers to their effective communication, and thereby, improve the quality of their services. Moreover, given the shift of educational programs for nurses from faceto-face education to electronic education, strategies are needed to ensure the quality of education, improve their understanding of educational materials, and promote their learning.

\section{Limitations}

One of the limitations of this study was the necessity of physical distancing during interviews. Moreover, appropriate face-to-face communication with participants was impossible due to the need for using face mask. The necessity to conduct interviews in the shortest possible time period to minimize the risk of COVID-19 transmission also required us to interview some participants in several short sessions and thereby, reduced our ability to collect coherent data in some cases.

\section{Authors' Contribution}

Study conception: El; Manuscript drafting: MHK. Data collection: EI and ZK; Data analysis: El and MHK; Manuscript editing and review: all authors.

\section{Conflict of Interests}

The authors declared no conflict of interests.

\section{Ethical Approval}

The Ethics Committee of Hormozgan University of Medical Sciences, Bandar Abbas, Iran, approved this study (code: IR.HUMS. REC.1399.180). Participants were informed about the study aim, reason for recording their interviews, voluntariness of participation, and confidentiality of their data. Informed consent was obtained from all participants. 
What does this paper contribute to the wider global clinical community?

- Nurses experience dare straits, social life disturbances, and disturbance of peace during their communication in the COVID-19 pandemic.

- Nurses need strong family, peer, and managerial support and public appreciation in order to protect and promote their health during the COVID-19 pandemic.

- Psychoemotional support by managers is needed to boost nurses' hope, remove barriers to their effective communication, and improve the quality of their services.

\section{Funding/Support}

This study was funded by the Infectious and Tropical Diseases Research Center affiliated to the Research and Technology Administration of Hormozgan University of Medical Sciences, Bandar Abbas, Iran.

\section{Acknowledgement}

The Infectious and Tropical Diseases Research Center affiliated to the Research and Technology Administration of Hormozgan University of Medical Sciences, Bandar Abbas, Iran, financially supported this study. We would like to thank this administration as well as nurses who participated in this study.

\section{References}

1. Wanko Keutchafo EL, Kerr J, Jarvis MA. Evidence of nonverbal communication between nurses and older adults: a scoping review. BMC Nurs. 2020;19:53. doi: 10.1186/s12912-02000443-9.

2. Markides M. The importance of good communication between patient and health professionals. J Pediatr Hematol Oncol. 2011;33 Suppl 2:S123-5. doi: 10.1097/ MPH.0b013e318230e1e5.

3. Stirling B, Hatcher J, Harmston J. Communicating the changing role of a nurse in an epidemic: the example of the MERS-CoV outbreak in Saudi Arabia. J Healthc Commun. 2017;2(3):1-6. doi: 10.4172/2472-1654.100070.

4. Ornell F, Halpern SC, Kessler FHP, de Magalhães Narvaez JC. The impact of the COVID-19 pandemic on the mental health of healthcare professionals. Cad Saude Publica. 2020;36(4):e00063520. doi: 10.1590/0102-311×00063520.

5. Houchens N, Tipirneni R. Compassionate communication amid the COVID-19 pandemic. J Hosp Med. 2020;15(7):4379. doi: 10.12788/jhm.3472.

6. Morley G, Grady C, McCarthy J, Ulrich CM. COVID-19: ethical challenges for nurses. Hastings Cent Rep. 2020;50(3):35-9. doi: 10.1002/hast.1110.

7. Buheji M, Buhaid N. Nursing human factor during COVID-19 pandemic. Int J Nurs Sci. 2020;10(1):12-24. doi: 10.5923/j. nursing.20201001.02.

8. Maben J, Bridges J. COVID-19: supporting nurses' psychological and mental health. J Clin Nurs. 2020;29(1516):2742-50. doi: 10.1111/jocn.15307.
9. World Health Organization (WHO). Risk Communication and Community Engagement Readiness and Response to Coronavirus Disease (COVID-19)! Interim Guidance, 19 March 2020. WHO; 2020. Available from: https://apps.who. int/iris/handle/10665/331513.

10. Fan PEM, Aloweni F, Lim SH, Ang SY, Perera K, Quek AH, et al. Needs and concerns of patients in isolation care unitslearnings from COVID-19: a reflection. World J Clin Cases. 2020;8(10):1763-6. doi: 10.12998/wjcc.v8.i10.1763.

11. McPeake J, Pattison N. COVID-19: moving beyond the pandemic. J Adv Nurs. 2020;76(10):2447-9. doi: 10.1111/ jan. 14438.

12. Shen X, Zou X, Zhong X, Yan J, Li L. Psychological stress of ICU nurses in the time of COVID-19. Crit Care. 2020;24(1):200. doi: 10.1186/s13054-020-02926-2.

13. Sun $N$, Wei $L$, Shi $S$, Jiao $D$, Song $R$, Ma L, et al. A qualitative study on the psychological experience of caregivers of COVID-19 patients. Am J Infect Control. 2020;48(6):592-8. doi: 10.1016/j.ajic.2020.03.018.

14. Yin X, Zeng L. A study on the psychological needs of nurses caring for patients with coronavirus disease 2019 from the perspective of the existence, relatedness, and growth theory. Int J Nurs Sci. 2020;7(2):157-60. doi: 10.1016/j. ijnss.2020.04.002.

15. Kim Y. Nurses' experiences of care for patients with Middle East respiratory syndrome-coronavirus in South Korea. Am J Infect Control. 2018;46(7):781-7. doi: 10.1016/j.ajic.2018.01.012.

16. Dowling M. From Husserl to van Manen. A review of different phenomenological approaches. Int J Nurs Stud. 2007;44(1):131-42. doi: 10.1016/j.ijnurstu.2005.11.026.

17. Polit DF, Beck CT. Essentials of Nursing Research: Appraising Evidence for Nursing Practice. 9th ed. Philadelphia: Wolters Kluwer Health; 2016. p. 704.

18. Van Manen M. Researching Lived Experience. 2nd ed. New York: Routledge; 2018.

19. Lai J, Ma S, Wang Y, Cai Z, Hu J, Wei N, et al. Factors associated with mental health outcomes among health care workers exposed to coronavirus disease 2019. JAMA Netw Open. 2020;3(3):e203976. doi: 10.1001/ jamanetworkopen.2020.3976.

20. Wang W, Tang J, Wei F. Updated understanding of the outbreak of 2019 novel coronavirus (2019-nCoV) in Wuhan, China. J Med Virol. 2020;92(4):441-7. doi: 10.1002/jmv.25689.

21. Zhu J, Sun L, Zhang L, Wang H, Fan A, Yang B, et al. Prevalence and influencing factors of anxiety and depression symptoms in the first-line medical staff fighting against COVID-19 in Gansu. Front Psychiatry. 2020;11:386. doi: 10.3389/ fpsyt.2020.00386.

22. Mast MS. On the importance of nonverbal communication in the physician-patient interaction. Patient Educ Couns. 2007;67(3):315-8. doi: 10.1016/j.pec.2007.03.005.

23. West CP, Dyrbye LN, Shanafelt TD. Physician burnout: contributors, consequences and solutions. J Intern Med. 2018;283(6):516-29. doi: 10.1111/joim.12752.

24. Liu C, Wang H, Zhou L, Xie H, Yang H, Yu Y, et al. Sources and symptoms of stress among nurses in the first Chinese anti-Ebola medical team during the Sierra Leone aid mission: a qualitative study. Int J Nurs Sci. 2019;6(2):187-91. doi: 10.1016/j.jinss.2019.03.007. 